Martin Bauer, MD

Rudolf Karch, PhD

Markus Zeitlinger, MD

Joan Liu, PhD

Matthias J. Koepp, PhD

Marie-Claude Asselin,

$\mathrm{PhD}$

Sanjay M. Sisodiya, PhD

Johannes A. Hainfellner,

MD

Wolfgang Wadsak, PhD

Markus Mitterhauser, $\mathrm{PhD}$

Markus Müller, MD

Ekaterina Pataraia, MD*

Oliver Langer, $\mathrm{PhD}^{*}$

Correspondence to

Dr. Langer:

oliver.langer@meduniwien.ac.at

Supplemental data at Neurology.org

\title{
In vivo P-glycoprotein function before and after epilepsy surgery OPEN
}

\section{ABSTRACT}

Objectives: To study the functional activity of the multidrug efflux transporter P-glycoprotein (Pgp) at the blood-brain barrier of patients with temporal lobe epilepsy using $(R)-\left[{ }^{11} C\right]$ verapamil (VPM)PET before and after temporal lobe surgery to assess whether postoperative changes in seizure frequency and antiepileptic drug load are associated with changes in Pgp function.

Methods: Seven patients with drug-resistant temporal lobe epilepsy underwent VPM-PET scans pre- and postsurgery. Patients were followed up for a median of 6 years (range 4-7) after surgery. Pgp immunoreactivity in surgically resected hippocampal specimens was determined with immunohistochemistry.

Results: Optimal surgical outcome, defined as seizure freedom and withdrawal of antiepileptic drugs, was associated with higher temporal lobe Pgp function before surgery, higher Pgppositive staining in surgically resected hippocampal specimens, and reduction in global Pgp function postoperatively, compared with nonoptimal surgery outcome.

Conclusions: The data from our pilot study suggest that Pgp overactivity in epilepsy is dynamic, and complete seizure control and elimination of antiepileptic medication is associated with reversal of overactivity, although these findings will require confirmation in a larger patient cohort. Neurology ${ }^{\circledR} 2014 ; 83: 1326-1331$

\section{GLOSSARY}

AED = antiepileptic drug; Pgp = P-glycoprotein; TL = temporal lobe; $\mathbf{T L E}=$ temporal lobe epilepsy; $\mathbf{V O I}=$ volume of interest; VPM $=(R)-\left[{ }^{11}\right.$ C]verapamil.

Approximately $30 \%$ of patients with epilepsy have inadequate seizure control despite taking antiepileptic drugs (AEDs). ${ }^{1}$ Surgery with removal of the anterior and mesial temporal lobe (TL) structures may result in favorable seizure outcome in $60 \%$ to $75 \%$ of patients, but longterm seizure freedom is achieved in only $50 \%$ with most of those patients still taking medication. $^{2}$ Biomarkers predicting outcome of epilepsy surgery would be of high clinical interest.

According to the transporter hypothesis of drug resistance, regional overactivity of multidrug efflux transporters, such as P-glycoprotein (Pgp), impedes access of substrate AEDs to their brain target sites, thereby rendering them ineffective. ${ }^{3}$ The hypothesis is well supported in some animal models of epilepsy. ${ }^{4,5}$ However, relevant in vivo human data are sparse. PET with the radiolabeled Pgp substrate $(R)-\left[{ }^{11} \mathrm{C}\right]$ verapamil $(\mathrm{VPM})$ can be used to noninvasively measure cerebral Pgp function. ${ }^{6}$ Two previous studies in patients with drug-resistant TL epilepsy (TLE) showed a trend for increased Pgp function in the ipsilateral TL compared with the contralateral side, ${ }^{7}$ and a significant bilateral increase in TL Pgp function compared with seizure-free patients. ${ }^{8}$ It remains unclear

\footnotetext{
*These authors contributed equally to this work.

From the Departments of Clinical Pharmacology (M.B., M.Z., M. Müller, O.L.) and Neurology (E.P.), Center for Medical Statistics, Informatics, and Intelligent Systems (R.K.), Institute of Neurology (J.A.H.), and Department of Biomedical Imaging and Image-guided Therapy, Division of Nuclear Medicine (W.W., M. Mitterhauser), Medical University of Vienna, Austria; Department of Clinical and Experimental Epilepsy (J.L., M.J.K., S.M.S.), UCL Institute of Neurology, London; Epilepsy Society (M.J.K., S.M.S.), Chalfont St Peter, Buckinghamshire; Institute for Population Health Wolfson Molecular Imaging Centre (M.-C.A.), University of Manchester, MAHSC, UK; and Health and Environment Department (O.L.), AIT Austrian Institute of Technology GmbH, Seibersdorf, Austria.

Go to Neurology.org for full disclosures. Funding information and disclosures deemed relevant by the authors, if any, are provided at the end of the article. The Article Processing Charge was paid by the Austrian Science Fund (FWF).

This is an open access article distributed under the terms of the Creative Commons Attribution-Noncommercial No Derivative 3.0 License, which permits downloading and sharing the work provided it is properly cited. The work cannot be changed in any way or used commercially.
} 
whether Pgp overactivity is static or changes with disease progression, and to what extent it depends on seizure activity or AED load. ${ }^{?}$

In the present study, we examined patients with TLE using VPM-PET before and after TL surgery in order to (1) assess whether postoperative changes in seizure frequency and AED load are associated with changes in Pgp function, and (2) correlate in vivo measurements of Pgp function with ex vivo immunohistochemistry. We hypothesized that optimal outcome after surgery would be associated with a reduction in Pgp activity.

METHODS Standard protocol approvals, registrations, and patient consents. The study was approved by the Ethics Committee of the Medical University of Vienna and was conducted as a pilot study. Written informed consent was obtained from all patients.

Patients. Seven patients ( 2 females; median age at time of first PET scan: 50 years, range 33-54) with drug-resistant $\mathrm{TLE}^{10}$ were included in this study after surgical treatment with either selective amygdalohippocampectomy $(\mathrm{n}=4)$ or anteromedial temporal lobectomy $(n=3)$. Patients were recruited from a group of 9 patients studied preoperatively with VPM-PET $; 2$ previous patients were not included because one patient was not available for follow-up after surgery and the other had no arterial input function for presurgery PET. A second VPM-PET scan was performed a median of 42 months (range 17-54) after surgery (see table for details). Surgery outcome in individual patients was ranked according to (1) complete seizure freedom after surgery, (2) type and number of seizures during follow-up period after surgery, and (3) intake of AEDs (figure e-1 on the Neurology ${ }^{\circledR}$ Web site at Neurology.org).

PET and MRI. Dynamic VPM-PET scans were acquired on an Advance PET scanner (GE Medical Systems, Waukesha, WI) as described before, ${ }^{7}$ with arterial blood sampled throughout. Radiolabeled metabolites of VPM were measured in discrete arterial blood samples using a previously described solid-phase extraction assay. ${ }^{7}$ For all patients, T1-weighted MRIs were acquired before and after surgery with an Achieva 3.0T scanner (Philips Medical Systems, Best, the Netherlands). The PET and MRIs were processed as described previously. ${ }^{7}$ PET data analysis was performed using the Hammersmith 3dimensional maximum probability atlas. ${ }^{7}$ Six TL volumes of interest (VOIs) (amygdala, parahippocampal gyrus, anterior temporal lobe, middle and inferior temporal gyrus, superior temporal gyrus, and posterior temporal lobe) and 2 extratemporal VOIs (superior parietal gyrus and cerebellum) were chosen for analysis. The hippocampus itself could not be analyzed because of spillover of radioactivity from the adjacent choroid plexus, which showed high VPM uptake.

Kinetic modeling of PET data was performed by using an arterial input function corrected for polar radiolabeled metabolites of VPM and a 1-tissue 2-rate constant compartment model to derive the transfer rate constant $K_{1}\left(\mathrm{~mL} / \mathrm{min} / \mathrm{cm}^{3}\right)$ of VPM from plasma into brain as an outcome parameter of Pgp function, with low $K_{1}$ indicating high Pgp function. ${ }^{7}$ To minimize the influence of radiolabeled metabolites of VPM on $K_{1}$ estimates, only the first 10 minutes of the PET data were considered for kinetic modeling. ${ }^{8}$
Immunohistochemistry. Brain tissue (hippocampus for all patients, and anterior-mesial parts of the TL of 3 patients) was removed during epilepsy surgery and fixed in $10 \%$ neutral buffered formalin immediately after surgery. For immunohistochemical staining, brain sections were prepared and probed with antibodies recognizing Pgp (clones, JSB1 1:400, C219 1:80, C494 1:1,000; Alexis Biochemicals, Lausen, Switzerland) followed by quantitative image assessment as published. ${ }^{8,11}$

Statistical analyses. Statistical testing was performed using Prism 5.0 software (GraphPad Software, La Jolla, CA). Data are presented as mean \pm SD for PET data and median (range) for demographic data. Friedman test with Dunn post hoc test was used when multiple groups were compared, and Wilcoxon matched-pairs signed rank test was used when 2 groups were compared. Correlations were assessed by calculating Spearman rank correlation coefficients $\left(r_{s}\right)$. A value of $p<0.05$ was considered statistically significant.

RESULTS Preoperative Pgp activity. In all patients, VPM- $K_{1}$ values from preoperative VPM-PET scans were lower in regions pathophysiologically connected to the epileptic focus (amygdala: $0.032 \pm 0.011$; superior temporal gyrus: $0.037 \pm 0.012$ ) compared with a reference region considered not to be involved in epileptogenic pathways (cerebellum: $0.045 \pm$ 0.012 ) (figure $1 \mathrm{~A})$. Moreover, VPM- $K_{1}$ was lower in amygdala than in posterior TL $(0.043 \pm 0.011)$. There were no differences between TL VPM- $K_{1}$ values ipsilaterally or contralaterally to the seizure focus.

Correlation of preoperative Pgp activity with Pgp expression. Pgp immunoreactivity in surgically resected hippocampal specimens was determined using Pgpspecific antibodies (figure 1B). The higher the Pgp immunoreactivity in surgically resected hippocampal specimens, the lower were the ipsilateral VPM- $K_{1}$ values for anterior TL $\left(r_{s}=-0.821, p=0.034\right.$; figure 1C) and superior temporal gyrus $\left(r_{s}=-0.857\right.$, $p=0.024)$.

Postoperative Pgp activity. Postoperative VPM- $K_{1}$ values were lower in superior temporal gyrus $(0.033 \pm$ $0.007)$ compared with cerebellum $(0.041 \pm 0.008)$ and the posterior TL $(0.041 \pm 0.009$; figure $1 \mathrm{D})$. The AED load was reduced in all patients, with 2 patients coming off all AEDs, but this did not affect peripheral metabolism of VPM; i.e., fractions of polar radiolabeled metabolites of VPM in plasma at $10 \mathrm{mi}$ nutes after injection were similar before $(0.140 \pm$ $0.039)$ and after surgery $(0.136 \pm 0.042, p=0.938)$.

Correlation of pre- and postoperative Pgp activity with postoperative outcome. Patients with better outcome had lower mean presurgical VPM- $K_{1}$ values in ipsilateral TL (higher Pgp function) than patients with poorer outcome $\left(r_{s}=0.837, p=0.024\right.$ ) (figure $2 \mathrm{~A}$ ). Patients with better outcome had high areas of Pgp immunopositive labeling in surgically resected hippocampal specimens, while patients with poorer outcome had 


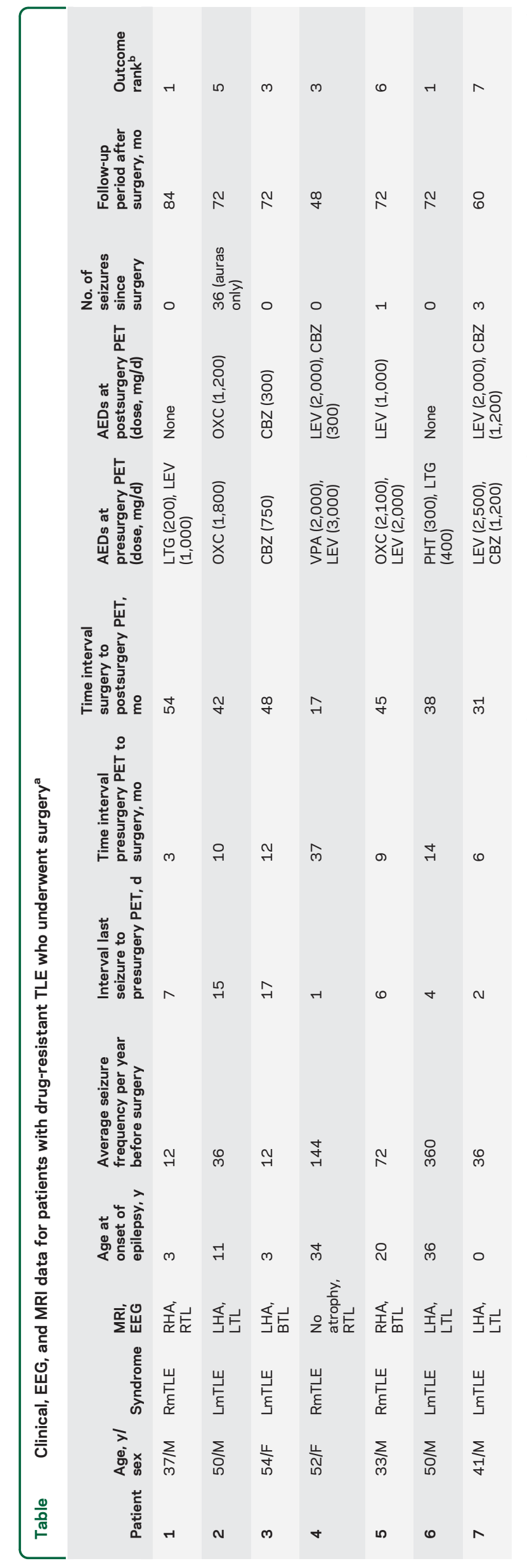

low Pgp immunoreactivity $\left(r_{s}=-0.782, p=0.048\right.$; figure $2 \mathrm{~B}$ ). In the 3 patients with best postoperative outcome (patients 1 and 6: seizure-free and without medication; patient 3: seizure-free and with very low AED load), VPM- $K_{1}$ values increased relative to preoperative PET scans in all brain VOIs studied, whereas $K_{1}$ values decreased in patients with worst outcome (figure 2C).

DISCUSSION In a longitudinal pilot VPM-PET study in patients with drug-resistant TLE before and after TL surgery with a median follow-up period after surgery of 6 years (range 4-7), we detected a "normalization" of Pgp activity in patients with long-lasting seizure freedom postoperatively. Our preoperative in vivo PET findings are supported by direct correlation with Pgp immunoreactivity in surgical specimens in the same patients. Furthermore, high Pgp activity in epileptogenic pathways preoperatively was associated with better postoperative outcome. Our longitudinal PET data suggest that Pgp overactivity is dynamic rather than static, and seizure freedom and reduction in AED load are associated with reversal of upregulation.

In line with the transporter hypothesis of drug resistance, we found significantly lower VPM- $K_{1}$ values in some TL brain regions close to the presumed epileptic focus (amygdala, superior temporal gyrus) as compared with a reference region outside the TL (cerebellum) or with a region more distant from the focus (posterior TL), pointing to Pgp overactivity in epileptogenic brain tissue. Consistent with previous findings, we show that Pgp overactivity was not restricted to the ipsilateral TL, but also extended to the contralateral side involved in epileptogenic pathways. ${ }^{8}$

After epilepsy surgery, patients with optimal surgery outcome defined as seizure freedom and withdrawal of AEDs had global VPM- $K_{1}$ increases relative to presurgery PET scans, suggestive of decreased Pgp function. This "return to normal" after successful epilepsy surgery is consistent with our recent report of higher VPM- $K_{1}$ values in pharmacosensitive compared with drug-refractory patients $^{8}$ and with findings in postmortem brain tissue, which showed almost no Pgp overexpression in the sclerotic hippocampus of patients with epilepsy who had entered terminal remission before death. ${ }^{11}$

A previous immunohistochemistry study showed higher Pgp expression in the TL white matter of patients with postoperative seizure relapse compared with postoperatively seizure-free patients, ${ }^{12}$ in keeping with our finding of increased Pgp activity postoperatively in patients with poorer outcome. Our preoperative in vivo PET data and ex vivo 
Figure 1 Pre- and postoperative Pgp activity and correlation with Pgp expression

A

B
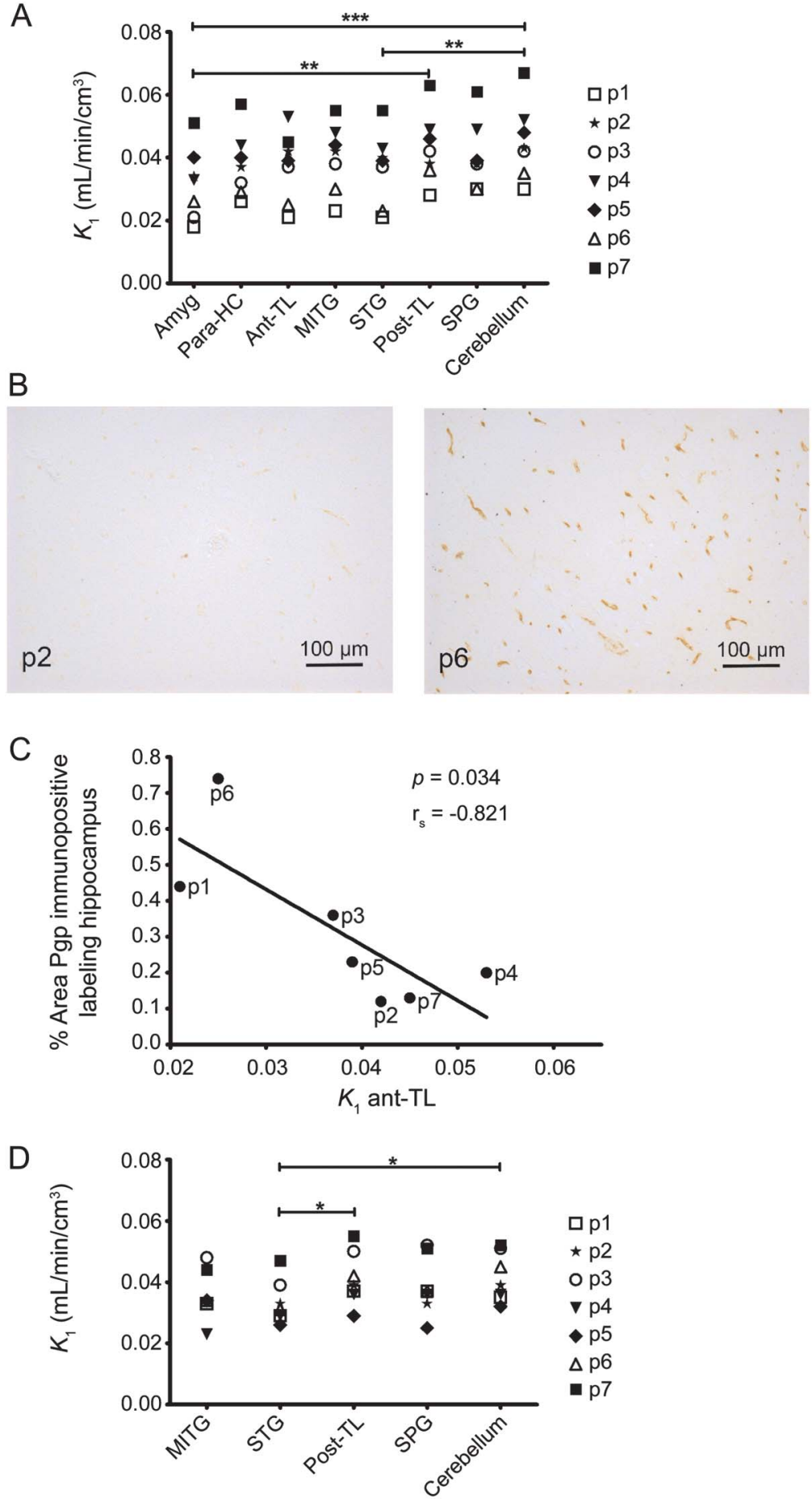

(A) VPM- $K_{1}$ values in different brain regions located ipsilateral to the seizure focus of individual patients before epilepsy surgery $(* * * p<0.001, * * p<0.01$; Friedman test with Dunn post hoc test). (B) Pgp immunopositive labeling in the surgically resected hippocampus of 2 patients. (C) Correlation of percentage area of Pgp immunopositive labeling in the surgically resected hippocampus with VPM- $K_{1}$ values in ipsilateral anterior $\mathrm{TL}\left(r_{\mathrm{s}}=\right.$ Spearman rank correlation coefficient). (D) VPM- $K_{1}$ values in different brain regions located ipsilateral to the seizure focus of individual patients after epilepsy surgery. Brain regions affected by surgery are not shown $(* p<0.05$; Friedman test with Dunn post hoc test). amyg = amygdala; ant$\mathrm{TL}=$ anterior temporal lobe; $\mathrm{MITG}=$ middle and inferior temporal gyrus; $\mathrm{p}=$ patient; para$\mathrm{HC}=$ parahippocampal gyrus; Pgp $=$ P-glycoprotein; post-TL $=$ posterior temporal lobe $\mathrm{SPG}=$ superior parietal gyrus; $\mathrm{STG}=$ superior temporal gyrus; $\mathrm{TL}=$ temporal lobe; VPM $=(R)-\left[{ }^{11}\right.$ Clverapamil. immunohistochemistry, however, suggest that Pgp overactivity in epileptogenic pathways and hippocampal overexpression are associated with optimal outcome after TL surgery. This discrepancy in predicting postoperative outcome might be explained by methodologic differences: we quantified hippocampal Pgp immunoreactivity, whereas Kwan et al. ${ }^{12}$ used semiquantitative approaches and found differences in Pgp immunoreactivity in TL white matter, but also a trend toward higher neuronal Pgp expression in postoperatively seizure-free patients. The latter finding is in keeping with our in vivo PET finding of Pgp overactivity in TL gray matter as a predictor of optimal outcome.

Our pilot study was not designed to assess the predictive ability of VPM-PET as biomarker of surgery outcome; this should be tested prospectively in a larger cohort, and integrated with other known risk factors for less favorable outcome, such as psychiatric comorbidities and incompleteness of resection. Moreover, our study cannot distinguish between the effects of seizure cessation from dose decrease or discontinuation of AEDs on postoperative Pgp function. Our cohort was too small to statistically compare subgroups of patients, e.g., seizure-free $(\mathrm{n}=4)$ vs not seizure-free $(\mathrm{n}=3)$ patients, or seizure-free patients without $\operatorname{AEDs}(n=2)$ vs seizure-free patients on $\operatorname{AEDs}(n=2)$, with meaningful results. Alternatively, one could conduct a prospective study to leave the patients on their medication for the first year after surgery and to acquire 2 separate postsurgery PET scans, before and after the change in medication. However, our data suggest that AED exposure also affects Pgp activity, because our outcome ranking mainly based on seizure burden after surgery correlates with AED load. Another limitation of this study is that we were not able to obtain VPM- $K_{1}$ values in hippocampus, which would require the availability of a PET scanner with higher spatial resolution and advanced image reconstruction to overcome the spillover from the high VPM uptake in the adjacent choroid plexus. ${ }^{8}$

Our study cannot address whether Pgp overactivity is the cause of drug resistance in TLE, and thus "surgical" removal of this overactivity contributed to seizure freedom, or whether upregulation is a marker of seizure burden rather than a cause. ${ }^{9}$ In both scenarios, Pgp overactivity may reduce target concentrations of AEDs and thereby possibly contribute to drug resistance. Our study demonstrates that Pgp overactivity is not static, and thus could be therapeutically targeted by drugs that inhibit or downregulate Pgp to possibly prevent development of, or bring about reversal of, drug resistance mediated by Pgp.

Our data confirm previous findings that VPM-PET reflects Pgp function in vivo in patients with TLE, and 
Figure 2 Correlation of Pgp activity and Pgp expression with postoperative outcome

A

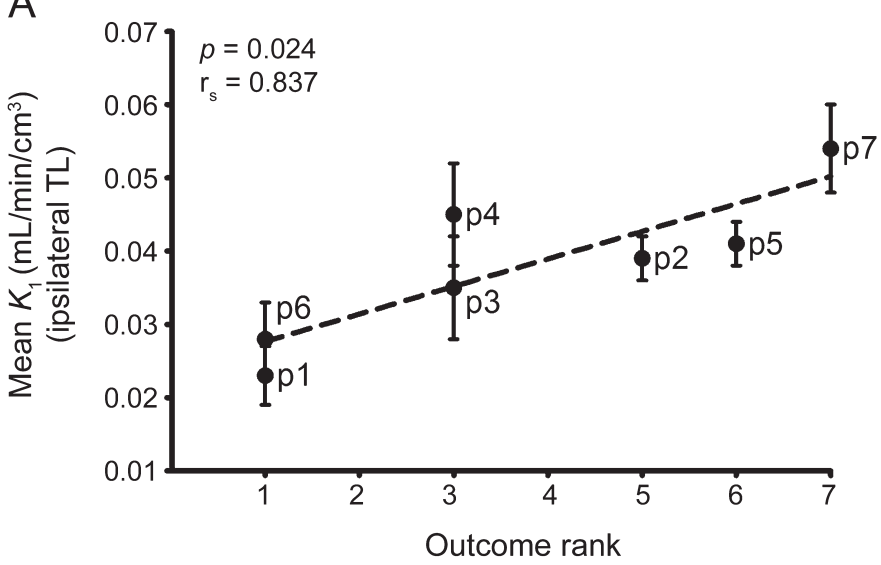

B

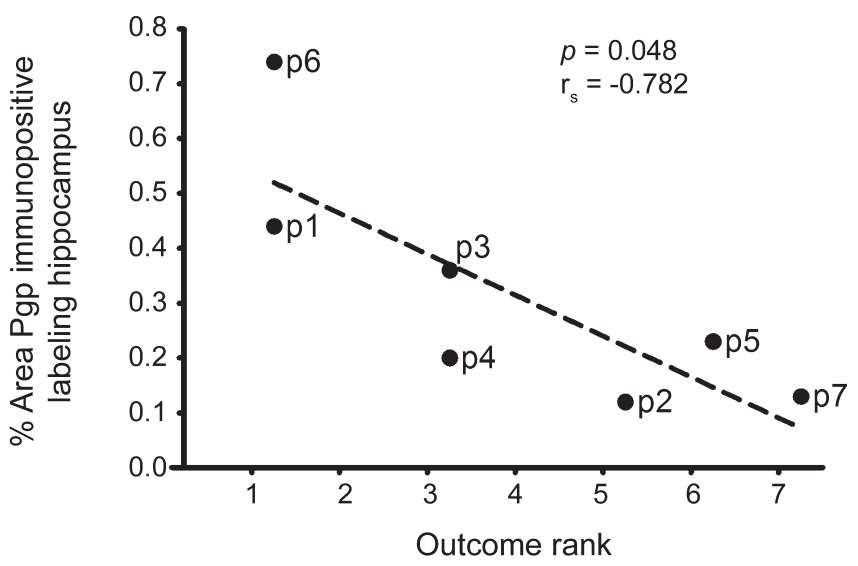

C

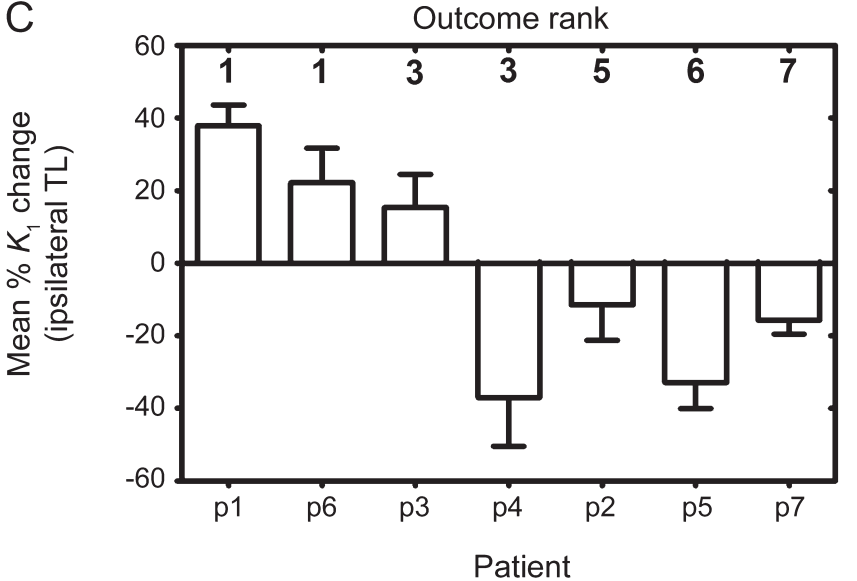

(A) Correlation of mean presurgical VPM- $K_{1}$ values in ipsilateral TL with surgery outcome rank in individual patients (see the methods section and figure e-1 for definition of surgery outcome rank order; $r_{s}=$ Spearman rank correlation coefficient). (B) Correlation of percentage area of Pgp immunopositive labeling in the surgically resected hippocampus with surgery outcome rank in individual patients. (C) Mean percent change in VPM- $K_{1}$ values across all studied TL regions located ipsilateral to the seizure focus in postsurgery PET scan relative to presurgery PET scan. Patients 3 and 4 were both assigned rank 3 , but the antiepileptic drug load of patient 3 was lower than that of patient 4. $p=$ patient; Pgp = P-glycoprotein; $\mathrm{TL}=$ temporal lobe; VPM $=(R)-\left[{ }^{11} \mathrm{C}\right]$ verapamil. that cerebral Pgp overactivity is associated with drug resistance in TLE. ${ }^{8}$ Our postoperative VPM-PET data extend previous findings, indicating a "normalization" of Pgp function in those patients who benefited most from surgery. Our findings also suggest that Pgp overactivity in the TL on preoperative VPM-PET might be indicative of optimal postoperative outcome.

\section{AUTHOR CONTRIBUTIONS}

Dr. Bauer: study concept or design, acquisition of data, analysis or interpretation of data, drafting/revising the manuscript for content. Dr. Karch: analysis or interpretation of data, statistical analysis, drafting/revising the manuscript for content. Dr. Zeitlinger: study concept and design, drafting/revising the manuscript for content. Dr. Liu: acquisition of data, analysis or interpretation of data, drafting/revising the manuscript for content. Dr. Koepp, Dr. Asselin, Dr. Sisodiya, and Dr. Hainfellner: analysis or interpretation of data, drafting/revising the manuscript for content. Dr. Wadsak and Dr. Mitterhauser: drafting/revising the manuscript for content, acquisition of data. Dr. Müller and Dr. Pataraia: study concept or design, analysis or interpretation of data, drafting/revising the manuscript for content. Dr. Langer: study concept or design, acquisition of data, analysis or interpretation of data, drafting/revising the manuscript for content.

\section{ACKNOWLEDGMENT}

The authors thank the staff of the PET center at the Department of Biomedical Imaging and Image-guided Therapy (Medical University of Vienna) and Research Nurse Edith Lackner (Department of Clinical Pharmacology, Medical University of Vienna) for their support in performing this study.

\section{STUDY FUNDING}

Supported by the European Community's Seventh Framework Program (grant 201380, to M.J.K, S.M.S., M.-C.A., M. Müller, and O.L.), the Austrian Science Fund (FWF) (grant F 3513-B20, to M. Müller and O.L., and KLI 139-B00, to M. Müller and O.L.), and the Department of Health's National Institute for Health Research Biomedical Research Centres funding scheme (to M.J.K. and S.M.S.)

\section{DISCLOSURE}

M. Bauer, R. Karch, M. Zeitlinger, and J. Liu report no disclosures relevant to the manuscript. M. Koepp is funded by the European Community's Seventh Framework Program (grant 201380, expired). Dr Koepp served on a scientific advisory board of GE Healthcare, and has received honoraria for lectures from UCB Pharma, Eisai, Novartis, and Desitin. M. Asselin is funded by the European Community's Seventh Framework Program (grant 201380, expired). S. Sisodiya is funded by the European Community's Seventh Framework Program (grant 201380, expired). Dr. Sisodiya served on a scientific advisory board for UCB Pharma, and has received institutional support or honoraria for lectures from UCB Pharma, GSK, and Eisai. J. Hainfellner, W. Wadsak, and M. Mitterhauser report no disclosures relevant to the manuscript. M. Müller is funded by the European Community's Seventh Framework Program (grant 201380, expired) and the Austrian Science Fund (FWF) (grants F 3513-B20 and KLI 139-B00). E. Pataraia reports no disclosures relevant to the manuscript. O. Langer is funded by the European Community's Seventh Framework Program (grant 201380, expired) and the Austrian Science Fund (FWF) (grants F 3513-B20 and KLI 139-B00). Go to Neurology.org for full disclosures.

Received December 20, 2013. Accepted in final form July 11, 2014.

\section{REFERENCES}

1. Kwan P, Brodie MJ. Epilepsy after the first drug fails: substitution or add-on? Seizure 2000;9:464-468.

2. Thom M, Mathern GW, Cross JH, Bertram EH. Mesial temporal lobe epilepsy: how do we improve surgical outcome? Ann Neurol 2010;68:424-434. 
3. Löscher W, Luna-Tortós C, Römermann K, Fedrowitz M. Do ATP-binding cassette transporters cause pharmacoresistance in epilepsy? Problems and approaches in determining which antiepileptic drugs are affected. Curr Pharm Des 2011;17:2808-2828.

4. van Vliet EA, van Schaik R, Edelbroek PM, et al. Inhibition of the multidrug transporter P-glycoprotein improves seizure control in phenytoin-treated chronic epileptic rats. Epilepsia 2006;47:672-680.

5. Brandt C, Bethmann K, Gastens AM, Löscher W. The multidrug transporter hypothesis of drug resistance in epilepsy: proof-of-principle in a rat model of temporal lobe epilepsy. Neurobiol Dis 2006;24:202-211.

6. Bauer M, Zeitlinger M, Karch R, et al. Pgp-mediated interaction between $(R)-\left[{ }^{11} \mathrm{C}\right]$ verapamil and tariquidar at the human blood-brain barrier: a comparison with rat data. Clin Pharmacol Ther 2012;91:227-233.

7. Langer O, Bauer M, Hammers A, et al. Pharmacoresistance in epilepsy: a pilot PET study with the P-glycoprotein substrate $R-\left[{ }^{11} \mathrm{C}\right]$ verapamil. Epilepsia 2007;48:1774-1784.
8. Feldmann M, Asselin MC, Liu J, et al. P-glycoprotein expression and function in patients with temporal lobe epilepsy: a case-control study. Lancet Neurol 2013;12:777-785.

9. French JA. P-glycoprotein expression and antiepileptic drug resistance. Lancet Neurol 2013;12:732-733.

10. Kwan P, Arzimanoglou A, Berg AT, et al. Definition of drug resistant epilepsy: consensus proposal by the ad hoc Task Force of the ILAE Commission on Therapeutic Strategies. Epilepsia 2010;51:1069-1077.

11. Liu JY, Thom M, Catarino CB, et al. Neuropathology of the blood-brain barrier and pharmaco-resistance in human epilepsy. Brain 2012;135:3115-3133.

12. Kwan P, Li HM, Al-Jufairi E, et al. Association between temporal lobe P-glycoprotein expression and seizure recurrence after surgery for pharmacoresistant temporal lobe epilepsy. Neurobiol Dis 2010;39:192-197.

13. Wieser HG, Blume WT, Fish D, et al. ILAE Commission Report: proposal for a new classification of outcome with respect to epileptic seizures following epilepsy surgery. Epilepsia 2001;42:282-286.

\section{Save These Dates for AAN CME Opportunities!}

Mark these dates on your calendar for exciting continuing education conferences by the American Academy of Neurology. Learn more at AAN.com/conferences.

\section{AAN Fall Conference}

- October 31-November 2, 2014, Las Vegas, NV, The Cosmopolitan of Las Vegas

AAN Annual Meeting

- April 18-25, 2015, Washington, DC, Walter E. Washington Convention Center

\section{BrainPAC}

BrainPAC is the American Academy of Neurology's federal political action committee.

- Since its inception, nearly 3,000 AAN members have contributed \$1,500,000 to BrainPAC.

- BrainPAC will contribute $\$ 600,000$ to candidates running for Congress in 2014.

- During the 2012 congressional campaign, 89 percent of candidates supported by BrainPAC won their elections.

BrainPAC supports both Democrats and Republicans who support issues important to the practice of neurology and the care of patients with neurologic conditions. US AAN members are invited to learn more at BrainPAC.org. 


\section{Neurology}

\section{In vivo P-glycoprotein function before and after epilepsy surgery}

Martin Bauer, Rudolf Karch, Markus Zeitlinger, et al.

Neurology 2014;83;1326-1331 Published Online before print September 3, 2014

DOI 10.1212/WNL.0000000000000858

This information is current as of September 3, 2014

Updated Information \&

Services

Supplementary Material

References

Subspecialty Collections

Permissions \& Licensing

Reprints including high resolution figures, can be found at:

http://n.neurology.org/content/83/15/1326.full

Supplementary material can be found at:

http://n.neurology.org/content/suppl/2014/09/03/WNL.0000000000000 858.DC1

This article cites 13 articles, 0 of which you can access for free at: http://n.neurology.org/content/83/15/1326.full\#ref-list-1

This article, along with others on similar topics, appears in the following collection(s):

All Epilepsy/Seizures

http://n.neurology.org/cgi/collection/all_epilepsy_seizures

Epilepsy surgery

http://n.neurology.org/cgi/collection/epilepsy_surgery_

Functional neuroimaging

http://n.neurology.org/cgi/collection/functional_neuroimaging

PET

http://n.neurology.org/cgi/collection/pet

PET in epilepsy

http://n.neurology.org/cgi/collection/pet_in_epilepsy

Information about reproducing this article in parts (figures,tables) or in its entirety can be found online at:

http://www.neurology.org/about/about_the_journal\#permissions

Information about ordering reprints can be found online:

http://n.neurology.org/subscribers/advertise

Neurology ${ }^{\circledR}$ is the official journal of the American Academy of Neurology. Published continuously since 1951, it is now a weekly with 48 issues per year. Copyright () 2014 American Academy of Neurology. All rights reserved. Print ISSN: 0028-3878. Online ISSN: 1526-632X.

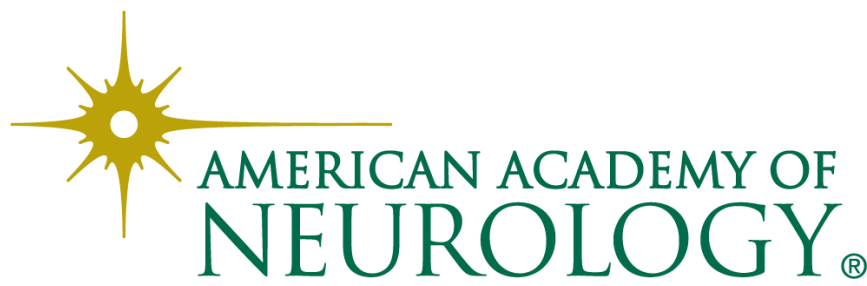

